\title{
Energetic electron precipitation and their atmospheric effect
}

\author{
Irina Mironova ${ }^{1,{ }^{*}}$, Miriam Sinnhuber ${ }^{2}$, and Eugene Rozanov $^{3,1}$ \\ ${ }^{1}$ Department of Physics of Earth, Faculty of Physics, Saint Petersburg State University, \\ Saint Petersburg, Russia \\ ${ }^{2}$ Karlsruhe Institute of Technology, Institute of Meteorology and Climate Research, Karlsruhe, \\ Germany \\ ${ }^{3}$ Physikalisch-Meteorologisches Observatorium World Radiation Center, Davos, IAC ETH, \\ Zurich, Switzerland
}

\begin{abstract}
Energetic particle precipitation induces ionization of the atmosphere which initiates a chain of reaction cycles affecting atmospheric composition and dynamics potentially down to surface weather systems. Ionization rates are retrieved based on yield functions or pre-calculated monoenergetic electron flux and energy spectra of precipitated energetic particles. Usually, information about energy spectra is obtained from satellites, balloons, and various ground-based observations. In all cases, some assumptions about spectral distribution for the entire energy range have to be made. As ionization rates are widely used in chemistry-climate models to estimate the atmospheric response to particle forcing, evaluation of the energy spectra is a key task in the solar-terrestrial studies. In this paper, it is shown that possible uncertainties of the ionization rates retrieval based on different spectral functions can lead to large disagreements in the ionization rates, with implications for the modelled response of atmospheric composition and dynamics to electron precipitation.
\end{abstract}

\section{Introduction}

Energetic electron and proton precipitation causes ionization of the Earth's atmosphere [1]. This leads to a chain of very fast ion chemistry reactions leading to the formation of reactive nitrogen and hydrogen, strengthening catalytic ozone loss cycles leading to ozone depletion, followed by changes in the atmospheric temperature and dynamics. These dynamical disturbances have been shown to propagate down even to tropospheric weather systems [2-4]. To study this so-called "geomagnetic forcing" of the climate system, chemistry-climate models have widely used ionization rates for the estimation of ozone destruction and the chemical composition of the atmosphere [e.g., 5-8].

\footnotetext{
* Corresponding author: $\underline{\text { i.a.mironova@spbu.ru }}$
} 
Retrieving ionization rates requires knowledge of the energy spectra and parameterization of ion production via ionization yield functions. The calculation of the ionization rates induced by energetic electron precipitation is usually based on the use of pre-calculated look-up tables containing ion production rates for precipitating monoenergetic electrons [9-11]. The ionization yield function at the atmospheric depth is the number of ion pairs created by one precipitating electron with the initial energy at the upper boundary of the atmosphere. The ionization rates (ion pairs $g^{-1} s^{-1}$ ) can be computed as $I(x)=\int Y(x, E) * F(E) d E$, where $Y(x, E)$ is yield functions, $F(E)$ is a flux of precipitating electrons at the top of atmosphere, $x$ - atmospheric depth and $E$ - energy of the considered particles. The limits of integration are defined by maximum and minimum energy of the considered electrons. The shape of the function describing electron energy distribution $F(E)$ at the top of atmosphere must be prescribed. Commonly used functions are exponential, exponential-law, Maxwellian and power-law spectral distribution. The form of the spectra function depends on energy range of precipitating particles. For instance, the exponential-law function is used for fitting energy of electron precipitation range covering by balloon-borne observations; Maxwellian spectral distribution describes well the auroral electrons and power-law spectral distribution is used for particles measured with the satellite instruments.

Energetic particle precipitation is monitored by low-earth orbit satellites and one of them is Polar Operational Environmental Satellites (POES) series of spacecraft with the MEPED instruments on board. MEPED measures energy-integrated electron fluxes in different channels for energies $>30 \mathrm{keV},>100 \mathrm{keV}$, and $>300 \mathrm{keV}$ [12]. Balloon observations of energetic particle precipitation in the atmosphere are an important independent source of information for the evaluation of satellite observed particles flux and energy widely used for retrieval ionization rates for climate models, extending the useful energy range from hundreds of $\mathrm{keV}$ to several $\mathrm{MeV}[13,14]$. The energy spectra and ionization due to precipitating electrons can be assessed using balloons. Balloon based devices register bremsstrahlung from energetic electron precipitation providing information on energy spectra of precipitating particles and further on the atmospheric ionization.

Recent analyses of atmospheric ionization rates provided for chemistry-climate model studies based on satellite observations of energy electron fluxes indicate uncertainties in the determination of atmospheric ionization rates [15-18].

The main goal of this paper is to demonstrate that the difference in the retrieval of ionization rates based on various fit functions covering the same energy range make a case of possible over- or under-estimation of ion productions which than can lead to disagreement between observation of chemical composition of the atmosphere and model results.

\section{Spectra functions and ionization rates of atmosphere}

The form of electron energy spectrum can be fitted using a function of exponential law $F(E)=A * \exp (-E / E 0)$, where $F(E)$ is the differential flux of precipitating electrons with kinetic energy $E, E O$ is the characteristic energy of precipitating electrons, $A$ is a parameter of the flux of incident electrons $\left(\mathrm{cm}^{-2} \mathrm{~s}^{-1} \mathrm{keV}^{-1}\right)$. An example of such type of spectra is presented in Figure 1a). Figure 1b) demonstrates spectra fitted with power-law function $F(E)=A * E^{k}$, where $F(E)$ is the power-law integral energy spectrum, $A$ is a parameter of the flux of incident electrons, and $k$ is the spectral gradient which can be varied from -1 to about -4 [19]. 
a) Exponential- law spectra

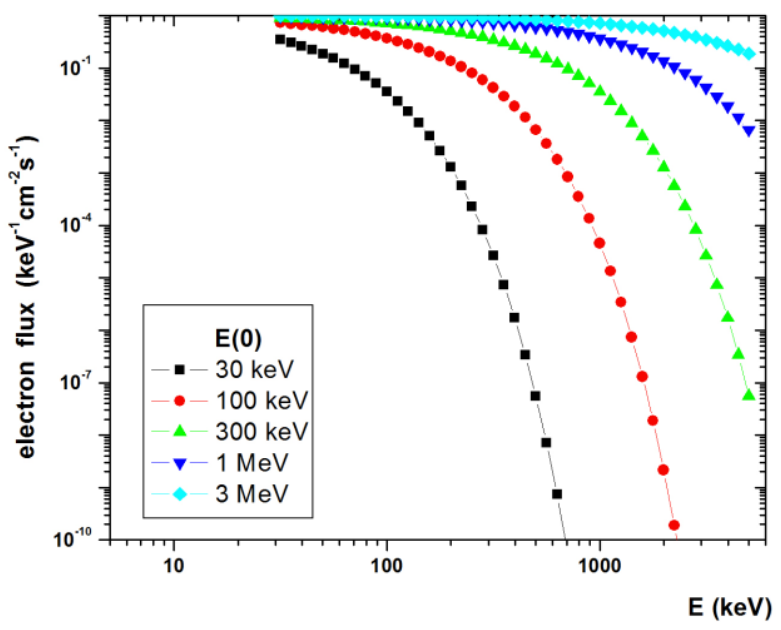

b)

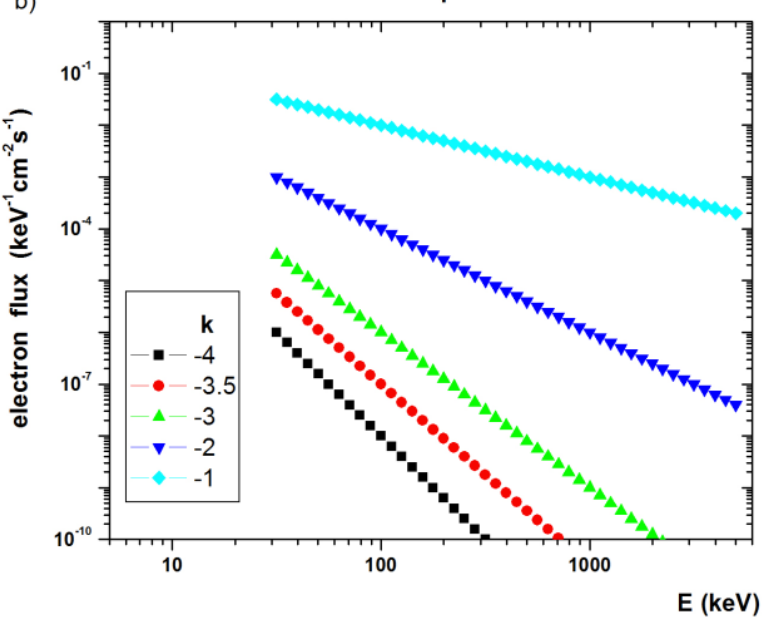

Fig. 1. Spectra functions of energetic electron precipitation. Electron flux is covering energy range from $30 \mathrm{keV}$ to $5 \mathrm{MeV}$. Energetic electron precipitation is considered as unit flux. a) An example of exponential law spectra for the characteristic energy of precipitating electrons $E 030 \mathrm{keV}, 100 \mathrm{keV}$, $300 \mathrm{keV}, 1 \mathrm{MeV}$ and $3 \mathrm{MeV}$. b) An example of power-law spectra for the $k$ spectral gradient $-4,-3.5$, $-3,-2,-1$.

Figure 1 shows spectra describing energetic electron precipitation by exponential and power-law functions. Electron flux is covering the energy range from $30 \mathrm{keV}$ to $5 \mathrm{MeV}$. The characteristic energy and spectral gradient cover all possible ranges to characterize various electron precipitation events observed by satellite and balloon-born sensors. Energetic electron precipitation is considered as unit flux of precipitating electrons. 
Ionization rates $I(x)$ are computed using a look-up table with ion production for isotropic precipitating monoenergetic electrons [6] and spectra proposed in Figure 1. The whole range of electron spectra includes energies from $30 \mathrm{keV}$ to $5 \mathrm{MeV}$ that defined as limits of integration. Figures $2 \mathrm{a}$ ) and $2 \mathrm{~b}$ ) present ionization rate profiles caused by the same characteristics of energetic electron precipitation but calculated using different spectra distributions. Ionization rates are normalized to corresponding values $I(x)$ at $10^{-3} \mathrm{~g} \mathrm{~cm}^{2}$.

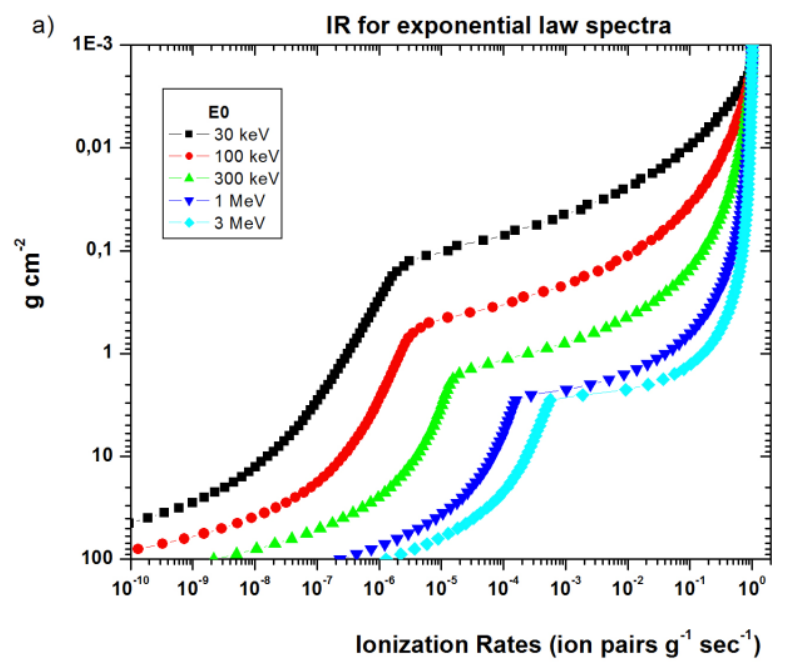

b)

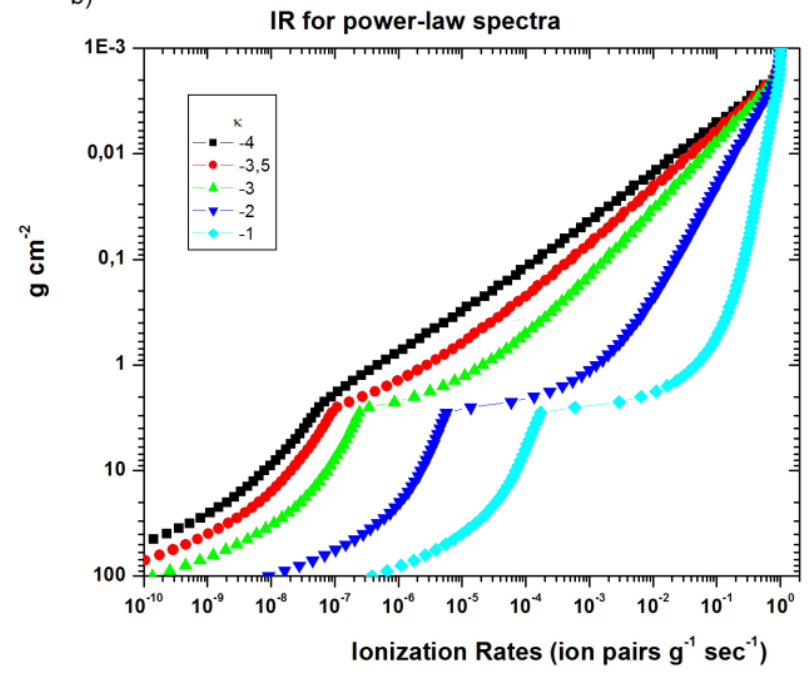

Fig. 2. Ionization rates (IR) are induced by unit flux of precipitating electrons with energies from 30 $\mathrm{keV}$ to $5 \mathrm{MeV}$. Ionization rates are calculated using exponential spectra (a) and power-law spectra (b) functions. 


\section{Impact on neutral atmosphere}

Atmospheric ionization rates are transferred to ions per $\mathrm{cm}^{-3} \mathrm{~s}^{-1}$ on a geopotential altitude grid and scaled with a constant factor $10^{11}$ leading to maximal ionization rates of slightly above $10^{4} \mathrm{~cm}^{-3} \mathrm{~s}^{-1}$ for the exponential power spectrum with characteristic energy of $3 \mathrm{MeV}$. Model calculations with the 1D stacked box model of the neutral and charged atmosphere ExoTIC [20] are carried out starting from a base neutral atmospheric composition provided by the HAMMONIA chemistry climate model for local noon on December 13, 2009 at $53^{\circ} \mathrm{N} / 0^{\circ} \mathrm{E}$, with a constant ionization rate forcing for one hour from 4-5 pm. Results at the end of the ionization period are shown in Fig. 3 compared to a model run without ionization for six ionization rate data provided in Figs. 2a) and 2b), respectively the highest, lowest, and middle value. Above $70 \mathrm{~km}$ altitude, formation of $\mathrm{NOx}$ and $\mathrm{OH}$ is observed in all model experiments, though absolute numbers vary by more than one order of magnitude. Ozone loss is observed in all model experiments down to $65 \mathrm{~km}$, with values varying from less than $0.1 \%$ to more than $50 \%$. Below these altitudes, increase of $\mathrm{NOx}, \mathrm{OH}$ and $\mathrm{HNO}_{3}$ as well as ozone loss are observed only in those model experiments with the highest ionization rates, while in the model experiments with lower rates, no response is observed below $\sim 60 \mathrm{~km}$ altitude.
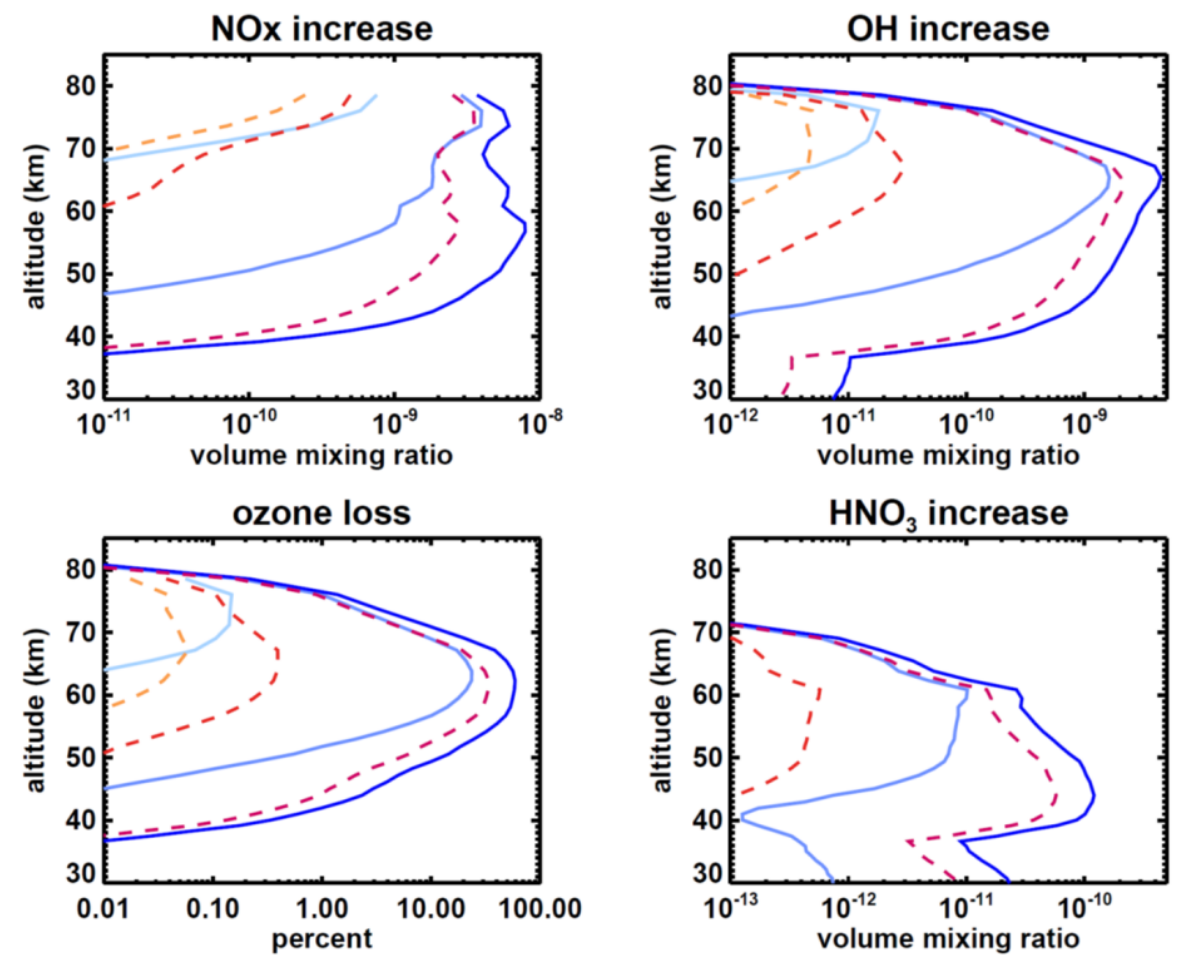

Fig. 3. Change of atmospheric constituents after one hour of constant ionization relative to a model run without ionization. Upper left $\mathrm{NOx}\left(\mathrm{NO}+\mathrm{NO}_{2}\right)$, upper right: $\mathrm{OH}$, lower left: ozone, lower right: $\mathrm{HNO}_{3}$. Solid lines: exponential-law spectra, characteristic energy of $30 \mathrm{keV}, 300 \mathrm{keV}$ and $3 \mathrm{MeV}$. Dashed lines: power law spectra, exponent $-4,-3$ and -1 . 


\section{Conclusion}

The difference in the retrieved ionization rates based on various spectra functions covering the same energy range makes a case of possible difference about several orders of magnitude in ionization rates at the same altitude. These can lead to differences of orders of magnitude in the atmospheric response as well. While in the upper mesosphere above 70 $\mathrm{km}$ altitude, an increase of $\mathrm{NOx}$ and $\mathrm{OH}$ as well as ozone loss are observed for all scenarios, below $\sim 60 \mathrm{~km}$, the different treatment of the spectra functions leads to a significant atmospheric impact only for scenarios with exponential power law and high characteristic energy, respectively scenarios with power-law spectra and small exponent.

\section{Acknowledgments}

This work is part of the German-Russian cooperation project "H-EPIC" funded by the Russian Foundation for Basic Research (RFBR project № 20-55-12020) and by the German Research Foundation DFG (grant SI 1088/7-1).

The work benefited from the discussions in the framework of the International Space Science Institute (ISSI and ISSI-BJ) team "Relativistic Electron Precipitation and its Atmospheric Effects".

ER acknowledges ISSI team "Space Weather Induced Direct Ionization Effects On The Ozone Layer".

\section{References}

1. Mironova, I. A., Aplin, K. L., Arnold, F., Bazilevskaya, G. A., Harrison, R. G., et al. Space Science Reviews, 194, 1-96, (2015)

2. Rozanov, E., L. Callis, M. Schlesinger, F. Yang, N. Andronova, and V. Zubov, Geophys.Res. Lett., 32, (2005)

3. Seppälä, A., Randall, C. E., Clilverd, M. A., Rozanov, E., and Rodger, C. J., J. Geophys. Res., 114, A10, (2009)

4. Maliniemi, V., Asikainen, T., and Mursula, K., J. Geophys. Res. 119, 16, 9752-9764, (2014)

5. Rozanov, E., Calisto, M., Egorova, T., Peter, T., \& Schmutz, W. Surveys in Geophysics, 33, 483-501, (2012)

6. Arsenovic P., E.Rozanov, A.Stenke, B.Funke, , J. Atmos. Sol.-Terr. Phys.. Volume 149, 180-190, (2016)

7. Matthes, K., Funke, B., Andersson, M. E., Barnard, L., Beer, J., Charbonneau, P., et al. Geos Model Development, 10, 2247-2302, (2017)

8. Sinnhuber M., H. Nieder, N. Wieters, Surv. Geophys. 33, 1281-1334 (2012)

9. Artamonov, A., Mironova, I., Kovaltsov, G., Mishev, et al., Adv, Space Res., 59(9), 2295-2300, (2017)

10. Artamonov, A. A., Mishev, A. L., \& Usoskin, I. G. J. Geophys. Res., 121, 1736-1743, (2016)

11. Fang, X., Randall, C. E., Lummerzheim, D., et al., Geophys. Res. Lett., 37, L22106, (2010)

12. Rodger, C.J., Clilverd, M.A., Green, J.C., Lam, M.M., J. Geophys. Res. Space Phys. $115 \mathrm{~A} 4,(2010)$

13. Makhmutov, V. S., G. A. Bazilevskaya, et al., J. Atmos. Solar-Terr. Phys., 149, 258- 
276, (2016)

14. Millan, R. M., R. P. Lin, D. M. Smith, and M. P. McCarthy, 2007. Geophys. Res. Lett. 34(10), L10101, (2007)

15. Wissing, J. M., Nieder, H., Yakovchouk, O. S., and Sinnhuber, M, J. Atmos. Sol.-Terr. Phys., 149, 191-206, (2016)

16. Nesse Tyssøy, H., M. I. Sandanger, L.-K. G. Ødegaard, et al., J. Geophys. Res., 120, 5693-5707, (2016)

17. Mironova, I. A., A. A. Artamonov, G. A. Bazilevskaya, E. V. Rozanov, et al., Geophys. Res. Lett., 46(2), 990-996, (2019)

18. Mironova I., G. Bazilevskaya, G. Kovaltsov, A. Artamonov, E. Rozanov, et al., Sci. Total Environ. 693, 133242, (2019)

19. Simon Wedlund, M., M. A. Clilverd, C. J. Rodger, et al., J. Geophys. Res. Space Physics, 119, 3961-3978, (2014)

20. Herbst, K., Grenfell, J.L., Sinnhuber, M., Rauer, H., Heber, B., Banjac, S., Scheucher, M., Schmidt, V., Gebauer, S., Lehmann, R., Schreier, F.,, A\&A, A101, (2019) 